\title{
Different absorption times of two absorb BVSs implanted in the same artery: insights into the mechanisms of late scaffold failure
}

\author{
Paweł Gąsior ${ }^{1}$, Radosław Parma ${ }^{1}$, Tomasz Roleder ${ }^{2}$, Wojciech Wojakowski ${ }^{1}$ \\ 'Division of Cardiology and Structural Heart Diseases, Medical University of Silesia, Katowice, Poland \\ ${ }^{2}$ Department of Cardiology, School of Medical Sciences, Medical University of Silesia, Katowice, Poland
}

We present an illustrative case of different absorption times of two absorb bioresorbable vascular scaffolds (BVS; Abbott Vascular, Santa Clara, CA, USA) implanted in the left anterior descending artery (LAD) (Fig. 1). It has been previously demonstrated that the appearance of polymeric scaffolds in optical coherence tomography (OCT) correlates with the integration and absorption processes [1]. In general, it is considered that the speed of bioresorption is homogeneous in coronary arteries because the primary mechanism of biodegradation is hydrolysis, which is a chemical process. Histological studies demonstrated complete bioresorption approximately 36 months after implantation, whereas in OCT strut footprint is visible as a black box for up to 36 months [2, 3]. In our case, initial predilation of the lesions before implantation of both scaffolds was performed. However, no intravascular imaging guidance was used during baseline procedures. The distal scaffold was implanted with subsequent postdilation 25 months ago in the medial LAD. The proximal scaffold was implanted with subsequent postdilation 38 months ago in the proximal LAD. Twelve months ago metallic drug eluting stent was implantated due to scaffold restenosis and two months ago balloon angioplasty due to thrombotic occlusion was performed. OCT images were recorded using the ILUMIEN PCI Optimisation System (St. Jude Medical, St. Paul, MN, USA). Paradoxically, OCT imaging demonstrated more advanced bioresorption and no restenosis of the distal scaffold (25 months old) compared to the proximal scaffold (38 months old) with visible struts and abnormal vessel wall remodelling. Interestingly, the bioresorption process in the whole artery was somehow accelerated and the process of the vessel wall integration of the distal scaffold was completed earlier than expected. On the other hand, the two re-interventions in the proximal scaffold might have resulted in fracture or embolisation, thus slowing down the bioresorption process.

\section{References}

1. Onuma Y, Serruys PW, Perkins LEL, et al. Intracoronary optical coherence tomography and histology at 1 month and 2, 3, and 4 years after implantation of everolimus-eluting bioresorbable vascular scaffolds in a porcine coronary artery model: an attempt to decipher the human optical coherence tomography images in the ABSORB trial. Circulation. 2010; 122(22): 2288-2300, doi: 10.1161/CIRCULATIONAHA.109.921528, indexed in Pubmed: 20975003.

2. Nakatani S, Ishibashi Y, Sotomi Y, et al. Bioresorption and Vessel Wall Integration of a Fully Bioresorbable Polymeric Everolimus-Eluting Scaffold: Optical Coherence Tomography, Intravascular Ultrasound, and Histological Study in a Porcine Model With 4-Year Follow-Up. JACC Cardiovasc Interv. 2016; 9(8): 838-851, doi: 10.1016/j.jcin.2016.01.030, indexed in Pubmed: 27101910.

3. Otsuka F, Pacheco E, Perkins LEL, et al. Long-term safety of an everolimus-eluting bioresorbable vascular scaffold and the cobalt-chromium XIENCE V stent in a porcine coronary artery model. Circ Cardiovasc Interv. 2014; 7(3): 330-342, doi: 10.1161/CIRCINTERVENTIONS.113.000990, indexed in Pubmed: 24895447.

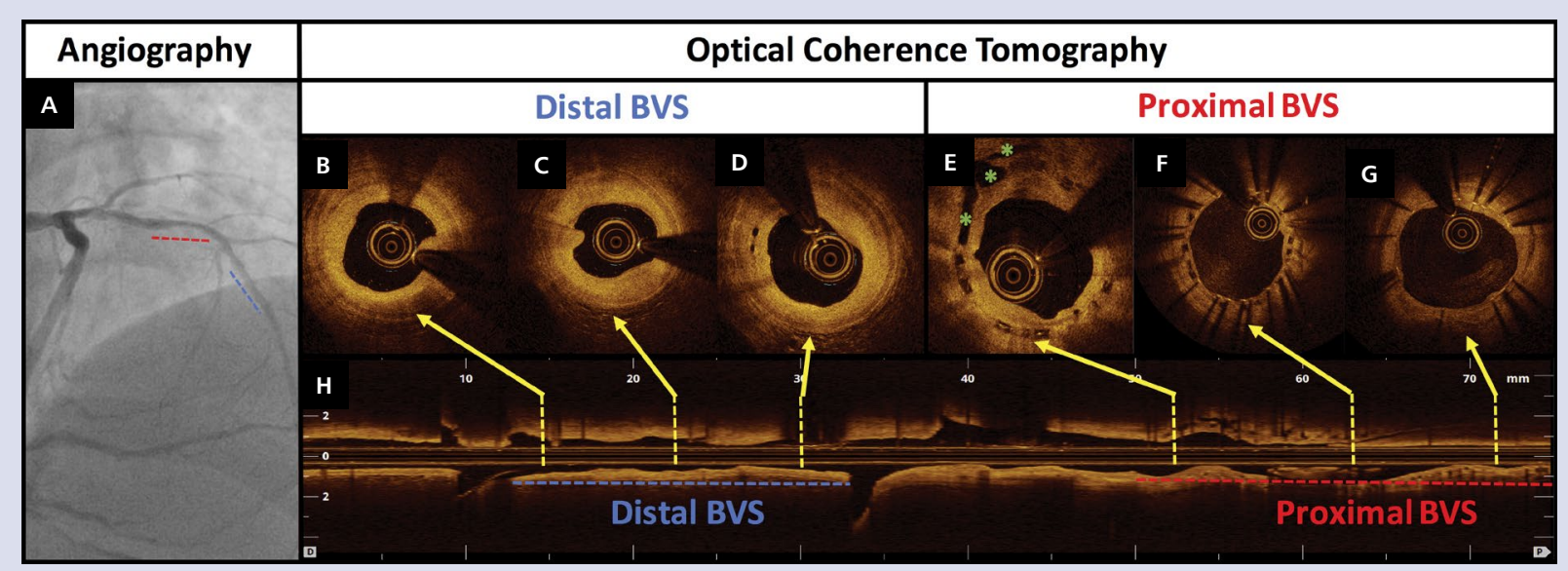

Figure 1. Angiography demonstrated no restenosis of the distal scaffold while in proximal scaffold a $50 \%$ obstruction was present (A). Optical coherence tomography (OCT) imaging of the distal bioresorbable vascular scaffold (BVS) revealed completed vessel wall integration process with only two visible struts and the appearance of a "golden tube" (B-D). OCT imaging of the distal segment of the proximal BVS revealed the presence of preserved strut cores and an empty area behind struts (green asterisk), which might suggest suboptimal implantation of the device resulting in underexpansion of the scaffold (E). Also, in the segment with overlapping BVS and drug eluting stent (DES), preserved struts were present (F). In the segment implanted only with DES mild neointimal proliferation was found $(\mathbf{G})$. Longitudinal OCT view is presented in figure $(\mathbf{H})$.

\section{Address for correspondence:}

Paweł Gąsior, MD, PhD, Division of Cardiology and Structural Heart Diseases, Medical University of Silesia, ul. Ziołowa 45, 40-635 Katowice, Poland, tel: +4832 25239 30, fax: +4832 25239 30, e-mail: p.m.gasior@gmail.com

Conflict of interest: none declared

Kardiologia Polska Copyright (c) Polish Cardiac Society 2018 